course and history of long term survivors and we describe characteristics and outcomes of all lung transplant recipients who have survived greater than 20 years at our centre.

Results Twenty-one (16.2\%) out of a possible total of 121 transplant patients survived at least 20 years with an overall median survival of 21.3 (range 20.1-24.9) years. The mean age at transplantation was $31.8 \pm 9.9$ years and $13(61.9 \%)$ were male. The most common indication for transplantation in the group was Cystic Fibrosis (33.3\%); heart-lung and bilateral lung transplant operations were equally the most commonly performed.

The median six-minute walk distance (6MWD) was $600 \mathrm{~m}$ (range $419-785 \mathrm{~m})$. The median time to the development of BOS was 9.7 years. At time of evaluation, $2(10 \%)$ patients had BOS score 0, 3 (14\%) BOS 1, 6 (29\%) BOS 2 and $10(48 \%)$ BOS 3.

The total number of rejections requiring augmentation with corticosteroids was 30 episodes in 21 patients with an average of 1.4 (range 0-3) episodes per patient. Eighteen patients had at least one episode of rejection needing corticosteroids.

No patient developed symptomatic ischaemic heart disease; systemic hypertension was found in 19 (90.5\%) patients. Two $(9.5 \%)$ patients developed post-transplant lymphoproliferative disease. Four patients developed other malignancies, 3 of which were skin cancers and 1 renal cancer.

All 4 cases of diabetes post transplantation occurred in patients with Cystic Fibrosis. Eight patients required renal replacement therapy as a result of ciclosporin toxicity and four underwent renal transplantation.

Conclusion Twenty-one (16.2\%) patients in our cohort survived 20 years. Although nearly all patients developed an element of CLAD, exercise tolerance was preserved as judged by 6MWD. Hypertension was common and renal failure remained the most problematic complication of immunosuppression.

\section{P244 CHARACTERISTICS AND OUTCOMES IN LUNG TRANSPLANT RECIPIENTS AGED 65 AND OVER}

S Isse, R Hackett, D Thomas, P Catarino, S Tsui, IS Parmar. Papworth Hospital NHS Foundation Trust, Cambridge, UK

\subsection{6/thoraxjnl-2014-206260.372}

Rationale Lung transplantation has become an accepted treatment option in a select group of patients with end-stage lung disease. The current International Society for Heart and Lung Transplantation (ISHLT) guidelines suggest age above 65 is a relative contraindication. However, increasingly patients with COPD and IPF are being referred for consideration after this age. The outcomes in this group (above 65) are not well described. We have studied the characteristics and outcomes of patients aged 65 and over, offered lung transplantation in our institution.

Method Retrospective review from the transplant database and patient records. We examined the Age, Sex, Indication for transplant and cause of death in all transplant recipients aged 65 and over from 1991 to July 2013. As a control group we compared them with 50 single lung transplant recipients front the same era and institution who were under 65 . We used SPSS to generate the survival curves.

Results In total we had 19 lung transplant recipients aged 65 and over who all had a single lung transplant, with a mean survival of 1600 days post-transplant. 13/19 were male recipients.
Survival Functions

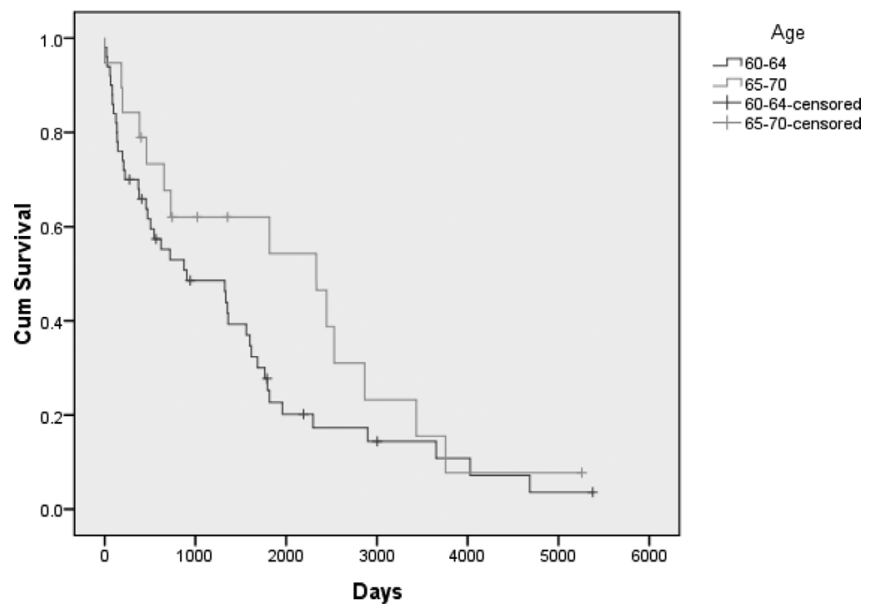

Abstracts P244 Figure 1 Survival Functions

The indication for transplantation was COPD and IPF in all. The cause of death was BOS in $9 / 14$, malignancy in $2 / 14$ and pulmonary embolism, stroke and bleeding in the others. When compared with 50 single lung transplant recipients aged 60-64, we did not find any statistically significant differences in survival ( $p$ value 0.158 ) (see figure 1), cause of death and reason for transplantation.

Conclusion We have shown from our limited data that patients aged 65 and over have very similar outcomes to their younger counterparts. Hence, age whilst still important should not be a deterring factor when referring patients for lung transplant assessment. It would also be important to examine longer term outcomes and complications such as rates of renal dysfunction, hypertension, rejection and admissions into hospital. As the number of patients aged 65 and older receiving lung transplant increases, we should be able to gather more effective data.

\section{P245 EVALUATION OF OUTCOMES OF ORAL RIBAVIRIN IN THE TREATMENT OF VIRAL LOWER RESPIRATORY TRACT INFECTION IN LUNG TRANSPLANT PATIENTS}

RJ Hackett, S Isse, J Parmar, D Thomas. Papworth Hospital, Cambridge, UK

\subsection{6/thoraxjnl-2014-206260.373}

Introduction Viral lower respiratory tract infections are common in lung transplant patients and contribute to the development of chronic rejection. Studies have highlighted the improvement in lung function and reduction in relative risk of chronic rejection in patients who are treated with appropriate anti-virals. Our study aimed to investigate the efficacy of three different routes of administration in patients with symptomatic declines in lung function and positive viral cultures.

Method Retrospective cohort study of viral respiratory tract infections treated with Ribavirin over a 5 year period was performed. Patients were divided in to 3 groups dependent on route of administration - Oral, Nebulised or Intravenous. Data was collected on patient demographics along with the indication for transplant, time since transplantation, pre and post treatment (68 weeks) lung function, viral cultures and details of any confounding factors such as prior rejection or concomitant bacterial infection were recorded. 
Abstract P245 Table 1

\begin{tabular}{llll}
\hline & Oral Ribavirin & Nebulised Ribavirin & Intravenous Ribavirin \\
\hline $\mathbf{n}$ & 11 & 13 & 10 \\
Mean Age & 52 & 48 & 47 \\
Type of transplant & 4 Bilateral Lung, 4 Single Lung, 3 Heart Lung & 9 Bilateral Lung, 1 Single Lung, 3 Heart Lung. & 7 Bilateral Lung, 1 Single Lung, 2 Heart Lung \\
Gender & $7 \mathrm{M} 4 \mathrm{~F}$ & $7 \mathrm{M} \mathrm{6F}$ & $5 \mathrm{M} \mathrm{5F}$ \\
Mean time since Transplantation (months) & 25.9 & 24.4 & 54.4 \\
Percentage change in FEV1 \pm SD & $96.4 \% \pm 9.9$ & $92.8 \% \pm 14.9$ & $93.3 \% \pm 10.5$ \\
$95 \%$ Confidence interval & $5.84(90.656 / 102.24)$ & $8.11(84.769 / 100.991)$ & $6.50(86.8 / 99.8)$ \\
\hline
\end{tabular}

Results 47 patients were identified. 12 patients were excluded due to insufficient clinical data. Of the remaining 34 patients, 11 were treated with Oral medication, 13 with Nebulised and 10 with Intravenous.

Conclusion Treatment with Ribavirin results in an improvement in lung function. In this small study it appears that there is no difference in lung function between the different routes of administration. Whilst the efficacy appears comparable oral treatment offers significant advantage to the patients and has benefits for treatment cost and bed occupancy.

\section{P246 LUNG TRANSPLANTATION FOR PATIENTS WITH IDIOPATHIC PULMONARY FIBROSIS AND ASYMPTOMATIC CORONARY ARTERY DISEASE}

P Riddell, K Redmond, D Eaton, L Nolke, SH Javadpour, D Healy, J McCarthy, JJ Egan. Mater Misericordiae University Hospital, Dublin, Ireland

\subsection{6/thoraxjnl-2014-206260.374}

Introduction and objectives Lung transplantation significantly improves the survival of patients with advanced idiopathic pulmonary fibrosis (IPF). Concurrent coronary artery disease (CAD) is a relative contraindication to transplantation and can limit access to this therapy. This is particularly relevant as a high prevalence of CAD has been reported in patients with $\mathrm{IPF}^{1}$ We sought to determine whether the presence of asymptomatic CAD impacted upon post-transplant survival.

Methods This retrospective study reviewed all patients who had undergone single lung transplantation for IPF at our centre, between May 2005 to April 2014. We compared post-surgical outcomes for patients with IPF who had an abnormal coronary angiogram (at the time of transplant listing), to those with normal angiography. Kaplan-Meier curves were created to study survival and univarate analysis performed using the Log-Rank score.

Results In this timeframe, 39 patients underwent lung transplantation for IPF, of which 22 patients $(56.4 \%)$ had abnormal coronary angiography. Eight of these patients had minor disease, 5 had $10-30 \%$ stenosis and 5 had $30-50 \%$ stenosis. One patient required coronary artery stenting prior to transplantation, but 3 patients with $\geq 70 \%$ stenosis had no inducible ischaemia on dobutamine stress testing and were managed conservatively. All patients had normal pre-operative left ventricular function.

The post-transplant survival of patients with IPF and CAD was $95.7 \%$ at 1 year and $70.1 \%$ at 5 years. There was no significant difference in survival $(\mathrm{p}=0.52)$ between the cohort with $\mathrm{CAD}$ and those with normal pre-operative coronary angiograms.

Of note, 1 patient required coronary artery stenting 18 months after transplantation (this patient had 30\% LAD stenosis at time of listing). No patients developed chronic arrhythmias, and no patients died as a result of cardiovascular disease.
Conclusion Although CAD remains a relative contraindication to transplantation, the effects of previous PCI or minor CAD are unknown and may be overstated. This finding is particularly relevant for patients with IPF, who may have an increased prevalence of CAD.

\section{REFERENCE}

1 Raghu G, Weycker D, Edelsberg J, Bradford WZ, Oster G. Incidence and prevalence of idiopathic pulmonary fibrosis. Am J Respir Crit Care Med. 2006:174 (7):810-6

\section{P247 MANAGEMENT OF AIRWAY STENOSIS AND BRONCHOMALACIA WITH BIODEGRADABLE STENTS AFTER LUNG TRANSPLANTATION. SINGLE INSTITUTION EXPERIENCE}

${ }^{1} S$ Gelvez-Zapata, ${ }^{1}$ A Wilkinson, 'D Thomas, ${ }^{2} \mathrm{M}$ Pittman, 'J Parmar. ${ }^{1}$ Papworth Hospital, Cambridge, UK, ${ }^{2}$ Basildon and Thurrock University Hospitals, Basildon, UK

\subsection{6/thoraxjnl-2014-206260.375}

Introduction Although the rate of airway stenosis following lung transplantation (LT) has reduced dramatically, it is still a significant cause of morbidity and mortality. Traditional self expanding metallic stents (SEMS) carry a high risk of bleeding and hyperplastic granulation tissue formation. Biodegradable stents (BS) present a potential alternative approach that could reduce these complications, though little is currently known about their effectiveness and safety.

Methods A retrospective analysis of our institutions use of 7 BS (polidioxanone) placed in 6 patients who presented bronchial stenoses after LT between December 2011 and January 2013. 2 patients with single (1 right and 1 left) and 4 with bilateral LT. The indications for placing the stents were anastomotic bronchomalacia in 3 cases and bronchial stenoses in 4 . The outcomes from these stents were compared with the last 10 patients who have SEMS.

Results Re-stenoses recurred in 3 cases, after 10, 6 and 4 months respectively; 2 responded to balloon dilatation and cryotherapy but 1 patient needed repeat stenting for restenosis. Stent migration occurred in 2 cases. No bleeding was reported. One patient died of obliterative bronchiolitis. The mean increase in FEV1 following treatment was a $312 \mathrm{ml}$ increase. Patients required an average of 4.3 (1-9) bronchoscopies following stent

Abstract P247 Table 1 Comparison of the outcomes following biodegradable and metallic stents insertion

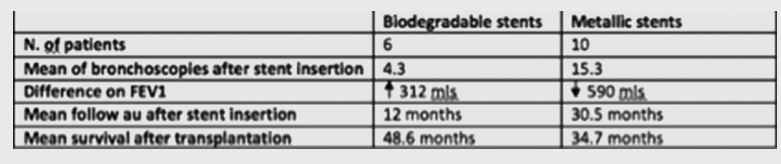

